\title{
Clasificación actual del carcinoma de pulmón. Consideraciones histológicas, inmunofenotípicas, moleculares y clínicas
}

Carlos Barrionuevo Cornejo* ${ }^{*}$; Daniela Dueñas Hancco ${ }^{2}$

\section{RESUMEN}

En los últimos años, el rápido desarrollo tecnológico y el mayor conocimiento de aspectos genéticos y moleculares en medicina han permitido un mejor enfoque en el entendimiento, diagnóstico y tratamiento de diversas enfermedades oncológicas. En relación con el carcinoma de pulmón, ha habido una significativa evolución desde sus primeras clasificaciones, puramente morfológicas, hasta la última del año 2015, en la que se integra información histológica, inmunofenotípica, genética molecular, clínica, y radiológica, lo que permite una mejor evaluación y manejo terapéutico de estos pacientes. En el presente artículo se hace un repaso desde las primeras clasificaciones del carcinoma de pulmón hasta la última, y se examinan los cambios más relevantes y la importancia de los hallazgos genéticos moleculares para un mejor enfoque clínico y terapéutico.

Palabras clave: Carcinoma; Pulmón; Mutación; Clasificación (Fuente: DeCS BIREME).

\section{Current classification of lung carcinoma. Histological, immunophenotypic, molecular and clinical considerations}

\begin{abstract}
In recent years, the rapid technological development and the better knowledge of genetic and molecular aspects in medicine have allowed a better approach to understand, diagnose and treat various oncological diseases. Regarding lung carcinoma, there has been a remarkable evolution from the first classifications, which were purely morphological, to the last one issued in 2015. This last classification includes histological, immunophenotypic, molecular genetics, clinical and radiological information, which allows a better evaluation and therapeutic management of these patients. In the present article, the first classifications of lung carcinoma to the last one are reviewed, and the most relevant changes and the importance of the molecular genetics findings are examined for a better clinical and therapeutic approach.
\end{abstract}

Keywords: Carcinoma; Lung; Mutation; Classification (Source: MeSH NLM).

1. Instituto Nacional de Enfermedades Neoplásicas, Departamento de Patología. Lima, Perú.

2. Hospital María Auxiliadora, Servicio de Patología. Lima, Perú.

*Autor corresponsal. 


\section{INTRODUCCIÓN}

En los últimos años, el conocimiento biológico de diversas enfermedades oncológicas se ha incrementado de forma acelerada, en gran parte, gracias al desarrollo tecnológico que ha permitido exitosas investigaciones en el campo genético molecular ${ }^{(1,2)}$.

Estos conocimientos han sido trasladados a la clínica (medicina traslacional), de tal manera que se ha determinado una estrecha correlación entre la biología molecular de las neoplasias malignas y el comportamiento clínico de estas, más aún, con la posibilidad de plantear terapias dirigidas al blanco, es decir, tratamientos personalizados. Los métodos diagnósticos de los tipos histológicos y moleculares de las diversas formas de cáncer se han beneficiado con el desarrollo tecnológico y la automatización de los laboratorios y, actualmente, se incluye la detección mutaciones en muestras de sangre periférica (biopsia líquida). Queda aún por resolver el alto costo de los denominados tratamientos biológicos, lo que requiere un esfuerzo colaborativo entre grupos de investigación, gobiernos e industria farmacéutica para crear plataformas de despistajes moleculares que consideren costos al alcance de la mayoría de la población ${ }^{(3-7)}$.

En cáncer pulmonar ha habido una serie de cambios epidemiológicos en las últimas décadas. Ha disminuido la frecuencia de carcinomas fuertemente asociados al tabaco (carcinoma escamoso y carcinoma de células pequeñas), y se han incrementado los adenocarcinomas no asociados a este elemento nocivo, de forma importante, en la población femenina, lo que está ligado con mutaciones genéticas descubiertas en los últimos años ${ }^{(8-10)}$.

En general, tanto en cáncer pulmonar como en otras neoplasias malignas, las clasificaciones histomorfológicas se están integrando a los conocimientos biológicos (genético moleculares e inmunofenotípicos) y clínicos para desarrollar clasificaciones con sentido práctico, que permitan predecir la evolución clínica y dar un tratamiento personalizado, eficaz y curativo. Esto es lo que está sucediendo vertiginosamente con el cáncer de pulmón, además, recientemente, se emplea la estimulación del propio sistema inmune como otra herramienta eficaz para el tratamiento del cáncer de células pequeñas y no pequeñas de pulmón (inmunoterapia) ${ }^{(9-15)}$.

\section{Antecedentes}

Una de las primeras clasificaciones del carcinoma de pulmón fue propuesta por Marchesani en 1924. En esta, se dividió al cáncer de pulmón en cuatro tipos principales, uno de los cuales fue el adenocarcinoma de células cilíndricas. Esta clasificación estuvo vigente por 25 años; pero luego de la Segunda Guerra Mundial, la frecuencia del cáncer de pulmón se incrementó notablemente, y se convirtió en un problema de salud pública. En 1967, la Organización Mundial de la Salud (OMS) publicó su primera clasificación luego de un amplio consenso entre patólogos expertos ${ }^{(16)}$, en la que se consideraron los siguientes tipos:

- Carcinoma epidermoide.

- Carcinoma de células pequeñas anaplásico.

- Adenocarcinoma (subtipos broncogénico, acinar, papilar y bronquioloalveolar).

- Carcinoma de células grandes anaplásico (subtipos sólidos con mucina, sólido sin mucina, de células gigantes y de células claras).

- Combinado (carcinoma epidermoidey adenocarcinoma).

Como puede advertirse por la nomenclatura, esta clasificación se basaba, fundamentalmente, en aspectos morfológicos de las neoplasias, aunque era claro que también existían diferencias de ubicación anatómica y curso clínico ${ }^{(17)}$. En 1981, la OMS publicó una nueva clasificación, basada en la anterior, pero con algunas modificaciones ${ }^{(18)}$. Se consideraron, dentro de los tumores epiteliales, 8 tipos principales (que incluían carcinoma escamoso, adenocarcinoma, carcinoma de células pequeñas y carcinoma de células grandes), 12 subtipos, y otros subgrupos recomendados, basados en el grado de diferenciación. Se describían, además, 70 tumores o seudotumores de la pleura o pulmón. Ya en esta clasificación se reconoce la correlación entre el curso clínico y los tipos histológicos principales de cáncer de pulmón; por ejemplo, el mejor pronóstico del carcinoma escamoso sobre el del adenocarcinoma o carcinoma de células grandes luego de cirugía radical o postradioterapia, o las respuestas del carcinoma de células pequeñas y adenocarcinoma a regímenes de quimioterapia específicos ${ }^{(17)}$. Sin embargo, se hicieron diversas observaciones en relación con las dificultades al emplear esta clasificación y su utilidad clínica. Estos problemas se enfocaban en la categorización de los casos (sobre todo en muestras citológicas), poca reproducibilidad entre patólogos, y la existencia de otros sistemas de clasificación como el de VALG (Veterans Adiministration Lung Cancer Chemotherapy Study Group), el WP-L (Working Party for Therapy of Lung Cancer) y el de la AFIP (Armed Forces Institute of Pathology) fueron algunas de estas observaciones ${ }^{(19,20)}$.

En 2004, luego de una reunión consenso en Lyon, la OMS en conjunto con la IASLC (International Association for the Study of Lung Cancer) publicó una clasificación oficial (21). El propósito fue, como en ocasiones previas, proveer criterios estándar para diagnóstico y nomenclatura del 
cáncer de pulmón. En esta ocasión se enfatizaron los criterios histológicos de las neoplasias epiteliales, aunque se reconoció la importancia de los exámenes auxiliares en patología, como la inmunohistoquímica, para una mejor categorización. Esta clasificación estuvo basada en una revisión de la versión de 1981 publicada en 1999 (22). Entre los principales cambios se incluyeron la revisión de los tumores neuroendocrinos y la mejor definición del carcinoma bronquioloalveolar, además de conceptos nuevos como los procesos preneoplásicos (hiperplasia adenomatosa atípica e hiperplasia atípica de células neuroendocrinas). La clasificación OMS 2004 define las siguientes neoplasias epiteliales malignas:

- Carcinoma de células escamosas y sus variantes (papilar, células claras, células pequeñas, basaloide).

- Carcinoma de células pequeñas y su variante combinada.

- Adenocarcinoma y sus tipos (acinar, papilar, bronquioloalveolar mucinoso y no mucinoso, mixto, sólido con producción de mucina, variantes diversas).

- Carcinoma de células grandes y sus variantes (neuroendocrino, combinado, basaloide, tipo linfoepitelioma, células claras, con fenotipo rabdoide).

- Carcinoma adenoescamoso.

- Carcinoma sarcomatoide y sus tipos (pleomórfico, de células fusiformes, de células gigantes, carcinosarcoma, blastoma pulmonar).

- Tumor carcinoide (típico y atípico).

- Tipo glándulas salivales (mucoepidermoide, adenoide quístico, epitelial-mioepitelial).

En cuanto a las lesiones preinvasivas, se definió la hiperplasia adenomatosa atípica (AAH, por sus siglas en inglés) como una proliferación localizada de células alveolares con atipia leve a moderada, sin inflamación ni fibrosis ${ }^{(8)}$ y con una imagen radiológica característica en vidrio esmerilado (23-26). La hiperplasia atípica de células neuroendocrinas (DIPNECH, por sus siglas en inglés) se definió como la presencia de pequeñas proliferaciones multifocales de células neuroendocrinas en el epitelio bronquiolar o bronquioloalveolar con posible compromiso del intersticio adyacente ${ }^{(26)}$. La importancia de esta lesión era la posibilidad de una progresión a tumores neuroendocrinos.

Bases de la clasificación OMS de carcinoma de pulmón 2015

Dado el importante y rápido avance del conocimiento de los mecanismos genéticos y moleculares en la patogénesis del cáncer de pulmón y su gran relevancia en el tratamiento de esta enfermedad, reflejado en diversas y múltiples publicaciones y reuniones académicas desde 2004, la OMS se propuso hacer una revisión de la clasificación sobre todo en relación con adenocarcinoma. Tres sociedades auspiciaron esta revisión, la ASLC (Asociation for the Study of Lung Cancer), la ERS (European Respiratory Society) y la ATS (American Thoracic Society). El equipo de investigadores estuvo liderado por William D. Travis, Elisabeth Brambilla y Masayuqui Noguchi ${ }^{(27)}$. Las recomendaciones se hicieron de acuerdo con los siguientes tópicos:

\section{Hallazgos moleculares}

La identificación de mutaciones, translocaciones y otras alteraciones genéticas que son "adictivas" para subgrupos de carcinomas pulmonares (generalmente, adenocarcinomas) ha permitido el diseño de terapias dirigidas con respuestas exitosas. Estas alteraciones constituyen conductores (drivers en inglés), usualmente tirosina quinasas constitutivamente activadas que afectan algunas rutas moleculares de señalización. Los genes alterados incluyen EGFR, KRAS, ALK, ROS, ERB2, BRAF, $M E T$, etc. ${ }^{(28-30)}$, y son la base actual de la clasificación molecular de los carcinomas pulmonares con fines de tratamientos dirigidos al blanco. Se ha enfatizado que este panorama de clasificación no reemplaza a la clasificación histológica si no, más bien, la enriquece ${ }^{(31)}$.

\section{Muestras pequeñas o citológicas}

Se han definido recomendaciones para diagnósticos en muestras pequeñas o citológicas, que incluyen los bloques celulares. Estas se basan en la importancia de definir un diagnóstico entre adenocarcinoma o carcinoma escamoso, dado que en el primer caso puede haber respuesta a inhibidores de tirosina quinasa (TKI) o a ciertas drogas como pemetexed y, el segundo caso, puede haber gran toxicidad por agentes como bevacizumab. Para esto, se recomienda el uso de ciertos marcadores de inmunohistoquímica (IHQ), aunque en forma limitada (TTF1 y NAPSIN-A para adenocarcinoma y P40 para carcinoma escamoso). Las recomendaciones son las siguientes:

a. Para muestras pequeñas o citológicas, se recomienda que los carcinomas de pulmón de células no pequeñas (NSCLC, por sus siglas en inglés) sean clasificados como adenocarcinoma o carcinoma de células escamosas en la medida que se posible.

b. En estos casos, se recomienda que el término NSCLC sea muy limitado, y que solo se utilice cuando no ha sido posible llegar a un diagnóstico específico por morfología o con el uso de técnicas como la IHQ.

\section{Recomendaciones para adenocarcinoma}

Para la nueva clasificación se hicieron las siguientes recomendaciones: 
a. No usar el término bronquioloalveolar (BAC, por sus siglas en inglés) debido a que sus criterios diagnósticos corresponden a adenocarcinoma in situ (AIS), denominación adecuada para estas lesiones.

b. Para adenocarcinoma solitario con patrón lepídico puro (antes $\mathrm{BAC}$ ) de $<3 \mathrm{~cm}$, se recomienda el usar el término AIS. La resección quirúrgica completa de estas lesiones son curativas al $100 \%$. La gran mayoría de AIS son no mucinosos.

c. Para adenocarcinoma solitario focal $(<3 \mathrm{~cm})$, con patrón lepídico predominante y foco invasivo microscópico de $0,5 \mathrm{~cm}$ o menos, se recomienda usar el nuevo término de adenocarcinoma mínimamente invasivo (MIA). Estos pacientes tienen cerca al $100 \%$ de sobrevida libre de enfermedad si se realiza la resección completa de la neoplasia. La mayoría de estos tumores son no mucinosos.

d. Para adenocarcinoma invasivo, se sugiere hacer la subtipificación de acuerdo con patrones histológicos, de manera semicuantitativa, en incrementos del $5 \%$, y escoger un patrón único predominante. Se recomienda que porcentajes de los subtipos sean reportados.

e. En pacientes con adenocarcinomas múltiple, se sugiere la subtipificación histológica en comparación con la muestra compleja heterogénea de patrones histológicos, para determinar si el tumor es metastásico o un primario aislado, metacrónico o sincrónico.

f. Para adenocarcinoma no mucinoso, previamente clasificado como subtipo mixto, en el cual el subtipo predominante consiste en un antes denominado BAC no mucinoso, se recomienda usar el término "adenocarcinoma predominantemente lepídico" (LPA, por sus siglas en inglés) y descontinuar el término "subtipo mixto".

g. En adenocarcinoma temprano, se recomienda adicionar como subtipo mayor al adenocarcinoma predominantemente micropapilar cuando sea el caso, ya que este es de pobre pronóstico.

h. Para los adenocarcinomas antes clasificados como BAC mucinoso, se recomienda separarlo del adenocarcinoma antes denominado BAC no mucinoso $y$, dependiendo de la extensión del crecimiento del crecimiento lepídico o invasivo, clasificarlos como AIS mucinoso, MIA mucinoso o, para tumores abiertamente invasivos, adenocarcinoma mucinoso invasivo.

De acuerdo a lo anterior, el adenocarcinoma in situ se define como una lesión en la cual el único patrón de crecimiento de las células tumorales es a lo largo de las paredes alveolares, sin invasión al intersticio. Los otros patrones de crecimiento son los relacionados al adenocarcinoma invasivo, que mantienen los tres patrones de la clasificación OMS 2004 (acinar, papilar, sólido) pero adiciona, como ya se mencionó, al micropapilar. Una gran proporción de adenocarcinomas resecados muestran, al menos, dos patrones diferentes (31).

En la nueva clasificación se reconoce el subtipo entérico, con características histomorfológicas e inmunohistoquímicas similares al primario intestinal y se han descartado los tipos "de células en anillo de sello" y "de células claras" pues corresponden más a variantes citológicas que pueden verse en los diversos subtipos de adenocarcinoma ${ }^{(32)}$.

\section{Carcinoma de células escamosas}

En este tipo de carcinoma, se han descartado los subtipos considerados en la clasificación anterior (papilar, células claras y células pequeñas), por su poca relevancia clínica y las dificultades en la definirlos adecuadamente. Se mantiene el subtipo basaloide debido a su impacto pronóstico desfavorable (libro WHO). Aunque no son frecuentes, se han identificado algunas alteraciones moleculares que podrían tener relevancia para tratamiento dirigido como son amplificación de FGFR1 (20\% de los casos) y PI3k (8\%), mutaciones de DDR2 (3\%) y de FGFR3 $(1 \%){ }^{(33,34)}$. Se ha remarcado la importancia del uso de la IHQ para diferenciarlo del adenocarcinoma en los casos poco diferenciados (P40, TTF1, NAPSIN-A) por las razones expuestas líneas arriba.

\section{Tumores neuroendocrinos}

Dada las diferencias clínicas, epidemiológicas, moleculares y genéticas entre los tumores carcinoides y los carcinomas neuroendocrinos, estas neoplasias se mantienen separadas. Se consideran dos tipos de carcinomas neuroendocrinos: de células pequeñas y de células grandes. Aunque ambas tienen ciertas semejanzas moleculares y epidemiológicas, aún no se justifica integrarlas en una sola entidad ${ }^{(35)}$. La hiperplasia de células neuroendocrinas pulmonar idiopática difusa se describe como una lesión preinvasiva ${ }^{(32)}$.

\section{Carcinoma de células grandes y carcinoma sarcomatoide \\ El carcinoma de células grandes pierde diferenciación morfológica e inmunofenotípica de linaje escamoso o glandular, de tal manera que corresponde a un carcinoma indiferenciado. El carcinoma sarcomatoide es un término genérico que engloba a lo carcinomas pleomórfico, fusocelular, de células gigantes, carcinosarcoma y blastoma pulmonar.}

\section{Carcinoma NUT}

Este raro carcinoma corresponde a una neoplasia poco diferenciada que se asocia a un rearreglo cromosómico en el gen NUT. Es una translocación entre este gen 
(NUTM1), ubicado en el cromosoma 15q14, y otros genes como BDR4 (cromosoma 19p13.1) en el $70 \%$ de los casos y BDR3 (cromosoma 9q34.2) en el $6 \%$ de los casos, o una pareja desconocida ( $24 \%$ de los casos). Se presenta en todas las edades, pero con mayor frecuencia en niños o adultos jóvenes ${ }^{(32,36)}$.

\section{CLASIFICACIÓN ACTUAL DE CARCINOMA DE PULMÓN OMS $2015^{(18)}$}

\section{ADENOCARCINOMA}

Neoplasia maligna epitelial con diferenciación glandular (Figuras 1 y 2 ).

a. Lepídico: constituido por neumocitos tipo II. Crece a lo largo de la superficie de las paredes alveolares, con área invasiva de más de $5 \mathrm{~mm}$.

b. Acinar: estructura glandular con lumen central rodeado por células tumorales.

c. Papilar: crecimiento papilar de células neoplásicas glandulares a lo largo de un core fibrovascular. d. Micropapilar: crecimiento en pequeños nidos papilares sin core fibrovascular.

e. Sólido: patrón predominante sin evidencia de patrón lepídico, acinar, papilar o micropapilar. Si el patrón sólido es del $100 \%$, deben haber, al menos, 5 o más células productoras de mucina por cada dos campos de alto poder, comprobadas con tinción de histoquímica.

f. Invasivo mucinoso: corresponde al antes denominado BAC mucinoso con morfología columnar o de células caliciformes con abundante mucina intracitoplasmática. Además del patrón lepídico, también puede presentarse con otros patrones.

g. Coloide: muestra abundante mucina reemplazando los espacios aéreos.

h. Fetal: estructura histológica semejante a tejido pulmonar fetal. Puede ser de alto o bajo grado.

i. Entérico: estructura histológica semejante al adenocarcinoma colorectal.
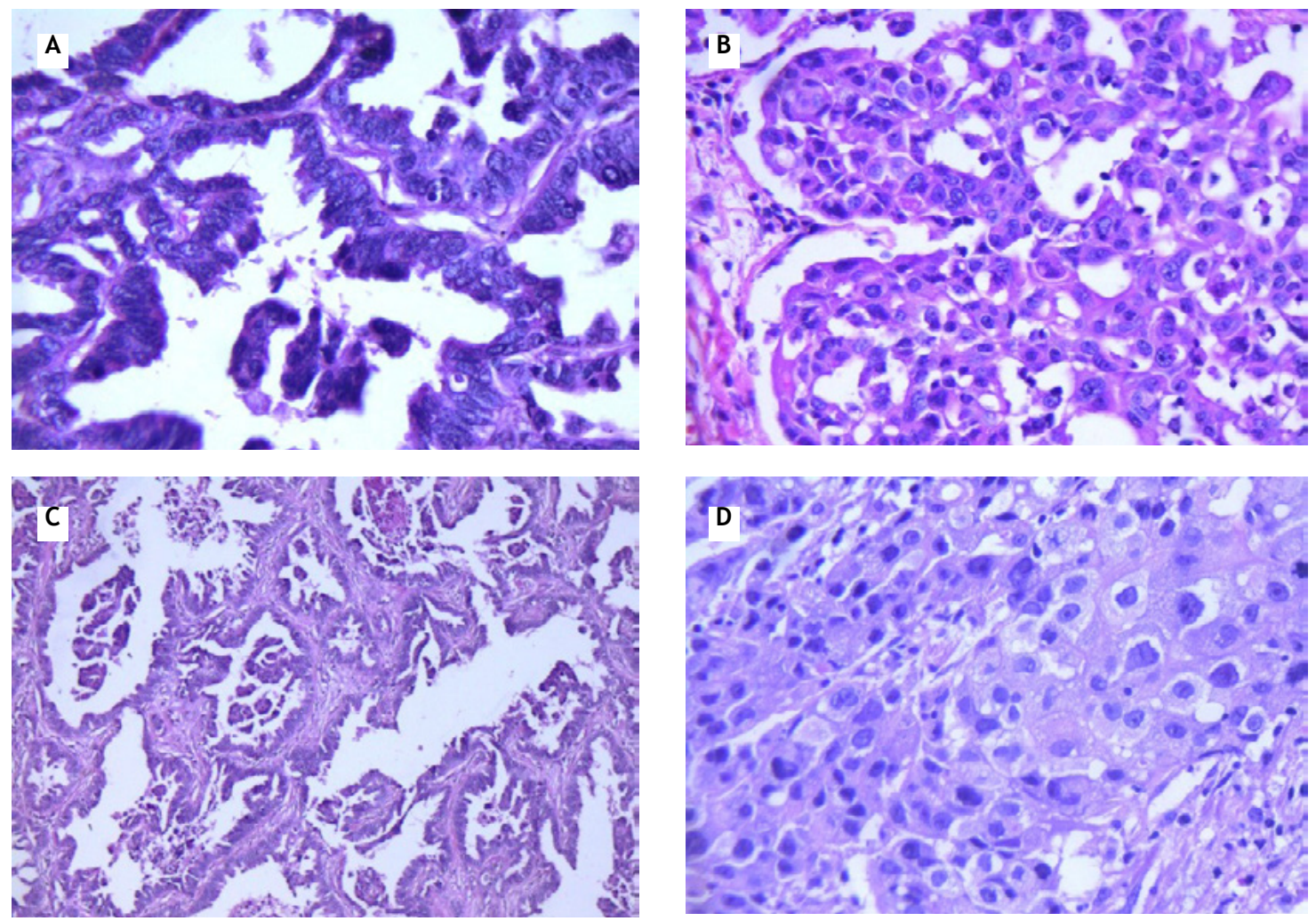

Figura 1: Adenocarcinoma. Tipos histológicos más frecuentes. A Patrón lepídico que muestra revestimiento de los alveolos por neumocitos neoplásicos (40X). B Patrón acinar medianamente diferenciado donde se advierte estructura tubular (40X). C Patrón papilar, donde se observan las proyecciones papilares intraluminales (10X). D Patrón sólido que no presenta túbulos, ni estructuras papilares 0 patrón lepídico (40X) 

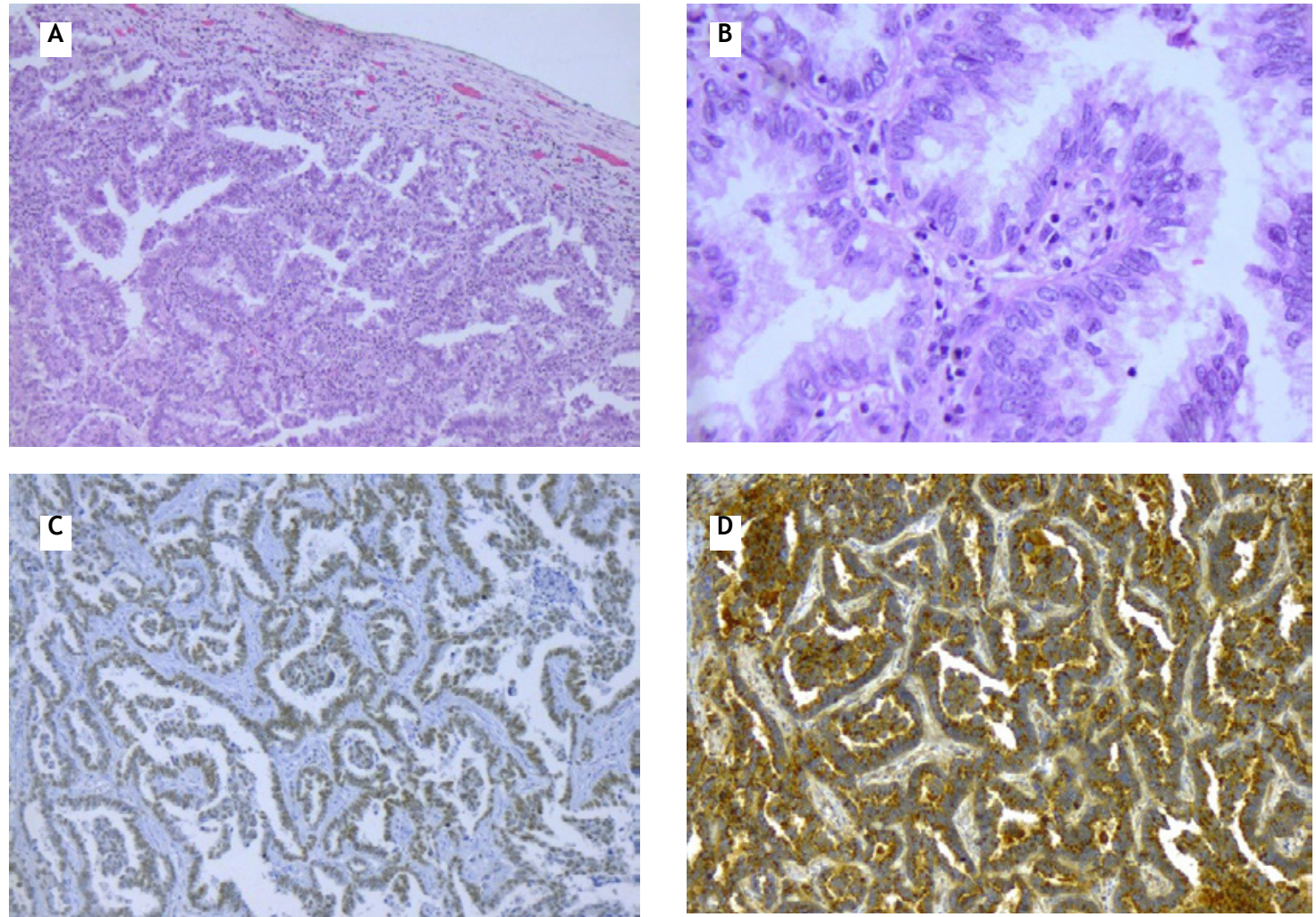

Figura 2: A Adenocarcinoma invasivo mucinoso, que evidencia mucina intracelular (10X). B Imagen a mayor aumento que muestra en detalle la mucina intracitoplasmática. C Adenocarcinoma papilar con expresión de TTF1 nuclear (10X). D El mismo caso anterior, expresando NAPSIN-A (40X)

j. Adenocarcinoma mínimamente invasivo: Adenocarcinoma solitario, de tamaño igual o menor de $3 \mathrm{~cm}$, con patrón lepídico, no mucinoso predominante y con invasión de hasta $5 \mathrm{~mm}$ en dimensión máxima.

k. Lesiones preinvasivas: Hiperplasia adenomatosa atípica: proliferación atípica localizada de neumocitos tipo II o células de Clara, de hasta $0,5 \mathrm{~cm}$ en dimensión máxima.

Adenocarcinoma in situ: adenocarcinoma localizado, usualmente no mucinoso, de hasta $3 \mathrm{~cm}$ en dimensión máxima, que crece a lo largo de estructuras alveolares preexistentes, en un patrón lepídico puro, sin invasión estromal ni vascular.

\section{CARCINOMA DE CÉLULAS ESCAMOSAS}

Neoplasia epitelial con presencia de queratinización o puentes intercelulares o con morfología indiferenciada, pero con marcadores de IHQ que indican diferenciación escamosa (Figura 3).

\section{a. Queratinizante \\ b. No queratinizante}

c.Basaloide: neoplasia poco diferenciada, con arquitectura lobular, con periferia en empalizada y pérdida de morfología escamosa, pero con marcadores de IHQ que indican dicho linaje. Puede haber casos con morfología escamosa queratinizante o no queratinizante, pero deben tener más de $50 \%$ de componente escamoso. Son de mal pronóstico.

d. Lesión preinvasiva: Carcinoma escamoso in situ: neoplasia de células escamosas, no invasiva, originada en el epitelio bronquial y que se origina en lesiones displásicas previas. 

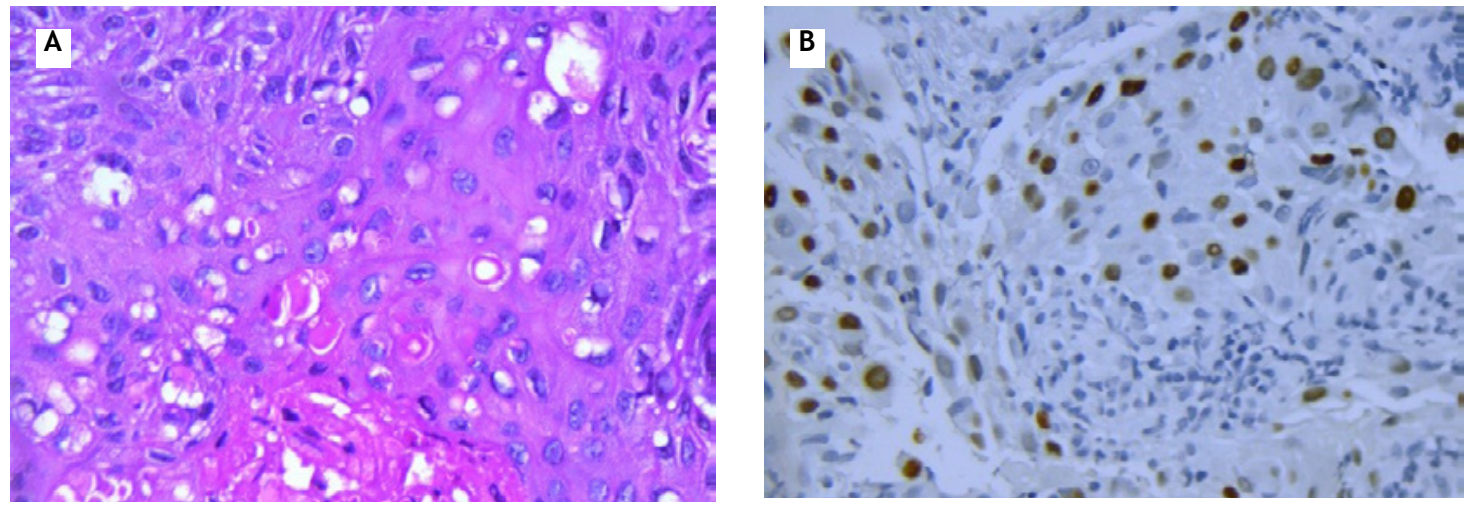

Figura 3: A. Carcinoma de células escamosas que muestra foco de queratinización (40X) B. El mismo caso anterior, que muestra expresión de P40.

\section{TUMORES NEUROENDOCRINOS}

a. Carcinoma de células pequeñas: neoplasia epitelial de células pequeñas, redondeadas, ovales o fusiformes, con núcleos de cromatina fina, nucleolo inconspicuo y escaso citoplasma. Usualmente, expresan gránulos neuroendocrinos. Puede ser puro o combinado con otro tipo de carcinoma no células pequeñas (Figura 4).

b. Carcinoma neuroendocrino de células grandes: neoplasia epitelial de células grandes, con morfología neuroendocrina (rosetas, empalizada), que expresa gránulos neuroendocrinos (Figura 5).

c. Carcinoide: neoplasia epitelial neuroendocrina que se divide en típico (menos de 2 mitosis por $2 \mathrm{~mm}^{2}$, sin necrosis) y atípico ( 2 a 10 mitosis por $2 \mathrm{~mm}^{2}$ o con focos de necrosis o ambos).

d. Lesión preinvasiva: Hiperplasia neuroendocrina pulmonar idiopática difusa: proliferación pulmonar de células neuroendocrinas que puede estar confinada a la mucosa, con o sin protrusión luminal, puede ocasionar invasión focal para formar "tumourlets" o puede convertirse a un tumor carcinoide.
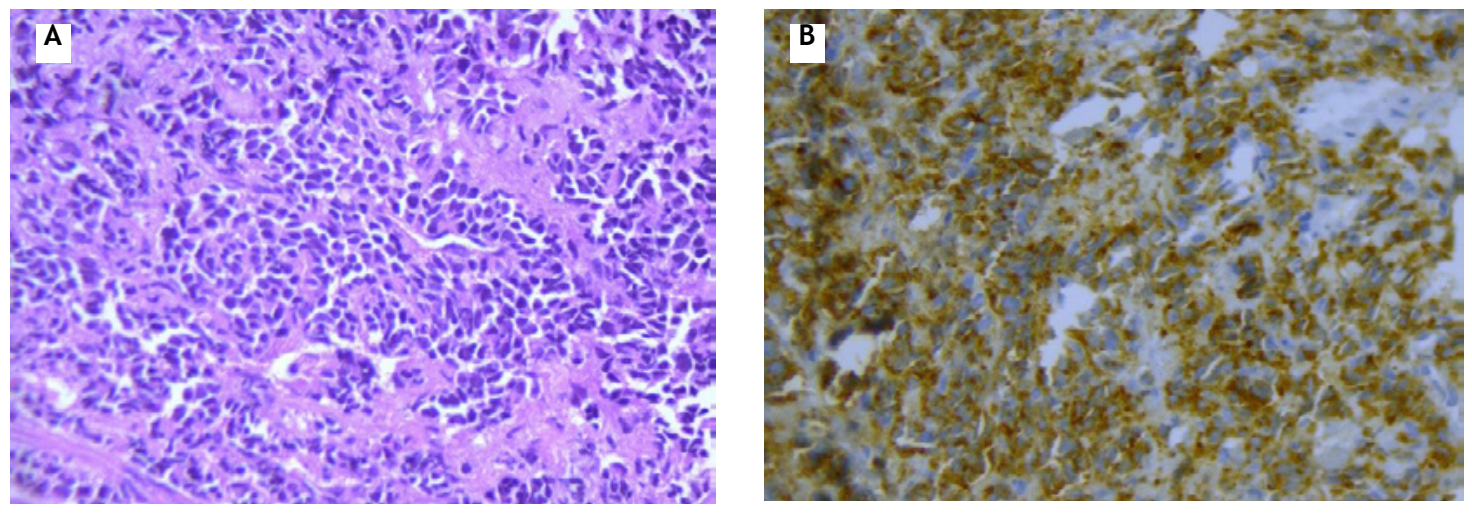

Figura 4: A Carcinoma de células pequeñas que muestra patrón trabecular (10X) B El mismo caso anterior, que muestra expresión de sinaptofisina (40X) 

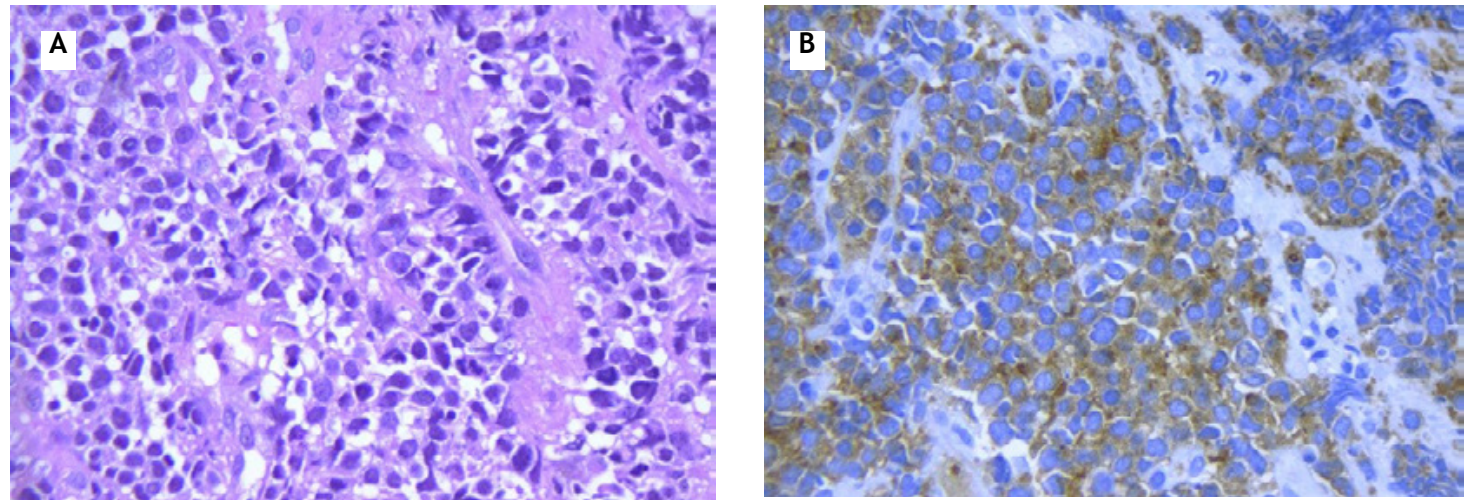

Figura 5: A Carcinoma neuroendocrino de células grandes que muestra patrón trabecular (40X). Nótese que algunas células son de tipo células claras B El mismo caso anterior, que muestra expresión de sinaptofisina (40X)

\section{CARCINOMA DE CÉLULAS GRANDES}

Neoplasia epitelial de células grandes sin diferenciación citohistológica o IHQ hacia adenocarcinoma, carcinoma escamoso o carcinoma de células pequeñas. Se divide en:

- Carcinoma de células grandes con marcadores de IHQ negativos (nulo).

- Carcinoma de células grandes con marcadores de IHQ poco claros.

- Carcinoma de células grandes en que no se han realizado marcadores de IHQ o tinciones simples.

\section{CARCINOMA ADENOESCAMOSO}

Neoplasia epitelial con componente de carcinoma escamoso y de adenocarcinoma. Debe tener al menos $10 \%$ de uno de los componentes.

\section{CARCINOMA PLEOMÓRFICO}

Carcinoma poco diferenciado que puede ser escamoso, adenocarcinoma o carcinoma de células pequeñas, con al menos $10 \%$ de células fusiformes o células gigantes.

\section{CARCINOMA DE CÉLULAS FUSIFORMES}

Constituido casi completamente por células fusiformes, sin componente diferenciado.

\section{CARCINOMA DE CÉLULAS GIGANTES}

Constituido casi completamente por células gigantes, que pueden ser multinucleadas, sin componente diferenciado.

\section{CARCINOSARCOMA}

Tumor maligno constituido por dos componentes: NSCLC, usualmente, carcinoma escamoso o adenocarcinoma, y sarcoma con elementos heterólogos (rabdomiosarcoma, condrosarcoma y osteosarcoma).

\section{BLASTOMA PULMONAR}

Tumor bifásico conformado por adenocarcinoma fetal (bajo de grado) con un estroma mesenquimal primitivo. Puede encontrarse foco de diferenciación mesenquimal específico (osteosarcoma, condrosarcoma o rabdomiosarcoma).

\section{CARCINOMA NO CLASIFICADO}

a.Carcinoma tipo linfoepitelioma: tipo poco frecuente de carcinoma poco diferenciado, intercalado con intenso infiltrado linfocítico, semejante al carcinoma faríngeo indiferenciado, con presencia del virus Epstein-Barr (EBV, por sus siglas en inglés) en el núcleo de las células neoplásicas.

b.Carcinoma NUT: carcinoma poco diferenciado, agresivo, caracterizado por rearreglo genético del gen NUT (nuclear protein in testis). Es extremadamente agresivo.

\section{CARCINOMA TIPO GLÁNDULA SALIVAL}

a.Carcinoma mucoepidermoide: constituido por células secretoras de mucina, células escamosas o escamoides y células tipo intermedias.

b.Carcinoma adenoide quístico: tumor maligno constituido por células epiteliales y células mioepiteliales. Presenta diversos patrones: tubular, cribiforme y sólido.

c. Carcinoma epitelial-mioepitelial: neoplasia epitelial maligna de bajo grado, con morfología bifásica. Presenta estructura ductal con una capa interna epitelial, rodeada por células mioepiteliales con morfología de células fusiformes, células claras o células plasmacitoides.

En conclusión, la nueva clasificación de carcinoma pulmonar representa un enfoque que integra las 
características histomorfológicas, inmunofenotípicas, clínicas, radiológicas y terapéuticas de estas neoplasias (37). Para graficar mejor lo dicho, transcribimos las palabras del Dr. Willian D Travis en las actas de la American Thoracic Societ publicadas en 2011: “En el campo de adenocarcinoma de pulmón, el mismo que progresa rápidamente, esta clasificación reúne muchos avances recientes que tendrán un impacto significativo en el diagnóstico y manejo de estos pacientes. Es necesario un enfoque de equipo multidisciplinario, particularmente para la gran mayoría de los pacientes con adenocarcinoma de pulmón que se presentan en etapa avanzada, donde se realizan y procesan muestras para citología y biopsias pequeñas, de tal manera que no solo se garantice diagnóstico histológico preciso, sino también un procesamiento molecular eficaz" ${ }^{27)}$.

\section{REFERENCIAS BIBLIOGRÁFICAS}

1. Ziogas DE, Katsios $\mathrm{C}$, Roukos $\mathrm{DH}$. From molecular traditional molecular biology to network oncology. Future Oncol. 2011; 7(2): 155-9.

2. Imran A, Qamar HY, Ali Q, Naeem H, Riaz M, Amin S, et al. Rol of molecular biology in cancer treatment: a review article. Iran J Public Health. 2017; 46(11): 1475-85.

3. Stahel R, Bogaerts J, Ciardiello F, De Ruysscher D, Dubsky $P$, Ducreux $M$, et al. Optimising translational oncology in clinical practice: strategies to accelerate progress in drug development. Cancer Treat Rev. 2015; 41(2): 129-35.

4. Fisher R, Pusztai L, Swanton C. Cancer heterogeneity: implications for targeted therapeutics. $\mathrm{Br}$ J Cancer. 2013; 108(3): 479-85.

5. O’Brien CP, Taylor SE, O'Leary JJ, Finn SP. Molecular testing in oncology: problems, pitfalls and progress. Lung Cancer. 2014; 83(3): 309-15.

6. Lawrence MS, Stojanov P, Polak P, Kryukov GV, Cibulskis K, Sivachenko A, et al. Mutational heterogeneity in cancer and the search for new cancer-associated genes. Nature. 2013; 499(7457): 214-8.

7. Crowley E, Di Nicolantonio F, Loupakis F, Bardelli A. Liquid biopsy: monitoring cancer-genetics in the blood. Nat Rev Clin Oncol. 2013; 10(8): 472-84.

8. De Groot PM, Wu CC, Carter BW, Munden RF. The epidemiology of lung cancer. Transl Lung Cancer Res. 2018; 7(3): 220-33.

9. Kashima J, Kitadai R, Okuma Y. Molecular and morphological profiling of lung cancer: a foundation for "next-generation" pathologists and oncologists. Cancers (Basel). 2019; 11(5).

10. Rudin CM, Poirier JT, Byers LA, Dive C, Dowlati A, George J, et al. Molecular subtypes of small cell lung cancer: a synthesis of human and mouse model data. Nat Rev Cancer. 2019; 19(7): 415.

11. Hoadley KA, Yau C, Wolf DM, Cherniack AD, Tamborero D, Ng $\mathrm{S}$, et al. Multiplatform analysis of 12 cancer types reveals molecular classification within and across tissues of origin. Cell. 2014; 158(4): 929-44.

12. Travis WD. Pathology of lung cancer. Clin Chest Med. 2011; 32(4): 669-92.

13. Jordan EJ, Kim HR, Arcila ME, Barron D, Chakravarty D, Gao J, et al. Prospective comprehensive molecular characterization of lung adenocarcinomas for efficient patient matching to approved and emerging therapies. Cancer Discov. 2017; 7(6): 596-609.

14. Gazdar AF, Bunn PA, Minna JD. Small-cell lung cancer: what we know, what we need to know and the path forward. Nat Rev Cancer. 2017; 17(12): 725-37.

15. Ott PA, Elez E, Hiret S, Kim DW, Morosky A, Saraf S, et al. Pembrolizumab in patients with extensive-stage small-cell lung cancer: results from the phase Ib KEYNOTE-028 study. J Clin Oncol. 2017; 35(34): 3823-9.

16. World Health Organization. Histological typing of lung tumours. 1st ed. Geneva: Kreyberg; 1967.

17. Lamb D. Histological classification of lung cancer. Thorax. 1984; 39(3): 161-5.

18. World Health Organization. Histological typing of lung tumours. 2nd ed. Geneva: Geneva; 1981.

19. Mathews MJ. Problems in morphology and behaviour of broncho-pulmonary malignant disease. In: Israel L, Chahinian AP, eds. Lung cancer-natural, prognosis and therapy. New York: Academic Press; 1976: 23-63.

20. Carter D, Eggleston JC. Tumours of the lower respiratory tract: atlas of tumour pathology. 2nd ed. Washington D. C.: Armed Forces Institute of Pathology; 1980.

21. Beasley MB, Brambilla E, Travis WD. The 2004 World Health Organization classification of lung tumors. Semin Roentgenol. 2005; 40(2): 90-7.

22. Travis WD, Colby TV, Corrin B, Shimosato Y, Brambilla E. Typing of lung and pleural tumors. 3a ed. Berlin: Springer; 1999.

23. Kawakami S, Sone S, Takashima S, Li F, Yang ZG, Maruyama Y, et al. Atypical adenomatous hyperplasia of the lung: correlation between high-resolution CT findings and histopathologic features. Eur Radiol. 2001; 11(5): 811-4.

24. Kushihashi T, Munechika H, Ri K, Kubota H, Ukisu R, Satoh S, et al. Bronchioloalveolar adenoma of the lung: CT-pathologic correlation. Radiology. 1994; 193(3): 789-93.

25. Kodama K, Higashiyama M, Yokouchi H, Takami K, Kuriyama K, Kusunoki Y, et al. Natural history of pure ground-glass opacity after long-term follow-up of more than 2 years. Ann Thorac Surg. 2002; 73(2): 386-92.

26. Nakajima R, Yokose T, Kakinuma R, Nagai K, Nishiwaki Y, Ochiai A. Localized pure ground-glass opacity on high-resolution CT: histologic characteristics. J Comput Assist Tomogr. 2002; 26(3): 323-9.

27. Travis WD, Brambilla E, Noguchi M, Nicholson AG, Geisinger $K R$, Yatabe $Y$, et al. International association for the study of lung cancer/american thoracic society/european respiratory society: international multidisciplinary classification of lung adenocarcinoma. J Thorac Oncol. 2011; 8(5): 381-5.

28. Weinstein IB. Cancer: addiction to oncogenes-the Achilles heel of cancer. Science. 2002; 297(5578): 63-4.

29. Shedden K, Taylor JM, Enkemann SA, Tsao MS, Yeatman TJ, Gerald WL, et al. Gene expression-based survival prediction in lung adenocarcinoma: a multi-site, blinded validation study. Nat Med. 2008; 14(8): 822-7.

30. Hayes DN, Monti S, Parmigiani G, Gilks CB, Naoki K, Bhattacharjee $A$, et al. Gene expression profiling reveals reproducible human lung adenocarcinoma subtypes in multiple independent patient cohorts. J Clin Oncol. 2006; 24(31): 5079-90.

31. Kerr KM. Classification of lung cancer: proposals for change?. 
Arch Pathol Lab Med. 2012; 136(10): 1190-3.

32. Travis WD, Brambilla E, Nicholson AG, Yatabe Y, Austin JHM, Beasley MB, et al. The 2015 World Health Organization Classification of lung tumors. J Thorac Oncol. 2015; 10(9): 1243-60.

33. Sos ML, Fischer S, Ullrich R, Peifer M, Heuckmann JM, Koker $M$, et al. Identifying genotype-dependent efficacy of single and combined PI3K- and MAPK-pathway inhibition in cancer. Proc Natl Acad Sci U S A. 2009; 106(43): 18351-6.

34. Weiss J, Sos ML, Seidel D, Peifer M, Zander T, Heuckmann JM, et al. Frequent and focal FGFR1 amplification associates with therapeutically tractable FGFR1 dependency in squamous cell lung cancer. Sci Transl Med. 2010; 2(62): 62-93.

35. Jones MH, Virtanen C, Honjoh D, Miyoshi T, Satoh Y, Okumura $\mathrm{S}$, et al. Two prognostically significant subtypes of high-grade lung neuroendocrine tumors independent of small-cell and large-cell neuroendocrine carcinomas identified by gene expression profiles. Lancet. 2004; 363(9411): 775-81.

36. Travis WD, Brambilla E, Nicholson AG, Yatabe Y, Austin JHM, Beasley MB, et al. The 2015 World Health Organization classification of lung tumors impact of genetic, clinical and radiologic advances since the 2004 classification. J Thorac Oncol. 2015; 10(9): 1243-60.

37. Travis WD, Brambilla E, Riely GJ. New pathologic classification of lung cancer: relevance for clinical practice and clinical trials. J Clin Oncol. 2013; 31(8): 992-1001.
Fuentes de financiamiento:

Este artículo ha sido financiado por los autores.

Conflicto de interés:

Los autores declaran no tener ningún conflicto de interés.

\section{Correspondencia:}

Carlos Barrionuevo Cornejo

Dirección: Avenida Angamos Este 2520. Surquillo. Lima, Perú. Teléfono: 2016500 (anexo 1429).

Correo electrónico: carlos.barrionuevo28@gmail.com

\section{Recibido: 24 de junio de 2019 \\ Evaluado: 10 de julio de 2019 \\ Aprobado: 25 de julio de 2019.}

( La revista. Publicado por Universidad de San Martín de Porres, Perú. (c) Bу Licencia de Creative Commons Artículo en acceso abierto bajo términos de Licencia Creative Commons Atribución 4.0 Internacional. (http://creativecommons.org/licenses/by/4.0/)

\section{ORCID iDs}

Carlos Barrionuevo Cornejo

Daniela Dueñas Hancco

https://orcid.org/0000-0003-2474-914X

https://orcid.org/0000-0001-7884-6784 